\title{
Fluid management for kidney transplantation: is it really about more or less?
}

\author{
Paula Perez Jimenez, MD · S. Joseph Kim, MD, PhD, MHS, MBA, FRCPC $\cdot$ Stuart A. McCluskey, MD, PhD, \\ FRCPCD
}

Received: 29 August 2021/Revised: 29 August 2021/Accepted: 31 August 2021/Published online: 16 November 2021

(C) Canadian Anesthesiologists' Society 2021

Annually, nearly 1,800 kidney transplants are conducted in Canada, yet, despite this effort, a similar number of people remain on the wait list for transplantation. ${ }^{1}$ This disparity in the supply and demand for organs is grossly underestimated by the actual shortfall, since less than one fifth of incident end-stage-kidney disease patients are referred for transplantation within 12 months of initiating dialysis. $^{2}$ Of the solid organ transplants, kidney transplantation is the most cost effective for the healthcare system because it offers the greatest benefits to the recipients in terms of longevity and quality of life. ${ }^{3}$ The need for transplantation and its benefits to the recipient and our healthcare system is a clear incentive for

P. Perez Jimenez, MD

Department of Anesthesiology and Pain Medicine, University of Toronto, Toronto, Canada

Department of Anesthesia and Pain Management, Toronto General Hospital, University Health Network, Toronto, ON, Canada

S. J. Kim, MD, PhD, MHS, MBA, FRCPC

Department of Medicine (Nephrology), University of Toronto,

Toronto, ON, Canada

Kidney Transplant Program, Toronto General Hospital,

University Health Network, Toronto, ON, Canada

S. A. McCluskey, MD, PhD, FRCPC ( $ه)$

Department of Anesthesiology and Pain Medicine, University of Toronto, Toronto, Canada

e-mail: stuart.mccluskey@uhn.on.ca

MultiOrgan Transplant Program, Toronto General Hospital, University Health Network, Eaton North 4-421, 200 Elizabeth Street, Toronto, ON M5G 2C4, Canada our efforts to improve both short- and long- term kidney graft function. ${ }^{4}$

In the immediate post-transplant period, delayed graft function (DGF) is a common occurrence, which has been strongly associated with length of hospital stay, postoperative complications, and long-term graft dysfunction. ${ }^{5}$ Delayed graft function is typically defined as the need for dialysis within the first week of transplantation, but there are additional measures of DGF based on the elimination of creatinine after transplantation or creatinine reduction ratios. These latter definitions may be more apt when the need for dialysis is rare (e.g., live donor kidney transplantation). ${ }^{6,7}$ Irrespective of the definition, DGF occurs in approximately $20-30 \%$ of deceased donor and $2-4 \%$ of live donor kidney transplants. While the etiology of DGF is multifactorial, intraoperative management is one potentially modifiable risk factor.

In this edition of the Journal, Kannan et al. compare the use of pulse pressure variability (PPV), measured using the arterial monitoring waveform (S/5 Avance ${ }^{\circledR}$ Anesthesia workstation, GE Healthcare/Datex-Ohmeda, Helsinki, Finland), with the central venous pressure (CVP) to guide fluid administration in kidney transplantation. ${ }^{8}$ They found a notable reduction in the mean (standard deviation) amount of fluid administered in the PPV group relative to the CVP group (1,346 [337] $\mathrm{mL}$ vs 1,901 [379] $\mathrm{mL})$. Clearly, finding this difference is determined as much by the control arm as by the treatment arm of their study. In the control group, CVP was used to guide fluid administration based on the method published by Othman et al., where fluid is restricted until the transplant graft is ready for vascular anastomosis. At this time, the CVP is increased to $15 \mathrm{~mm} \mathrm{Hg}$ with the administration of IV fluid. ${ }^{4}$ 
In the original study, this resulted in the administration of over $3 \mathrm{~L}$ of normal saline in the operating room. ${ }^{4}$

As the authors point out, the "traditional" use of CVP to guide fluid therapy is flawed because of "its limited reliability as a parameter of intravascular fluid status assessment". The anesthesiologist's objective is to optimize cardiac preload or, at the very least, ensure that cardiac performance or stroke volume is not limited while avoiding the administration of excessive amounts of fluids. The authors conclude that they accomplished this objective with less fluid, but that the study was inadequately powered to consider any clinically important outcomes. Nevertheless, the hypotheses generated by this study should be the basis for future investigations using either a larger population or a population at great risk of DGF.

When an arterial waveform is available either from arterial cannulation or non-invasive monitoring (e.g., ClearSight, Edwards Lifesciences, Irvine, CA, USA) the physiologic association between variability in pulse pressure and the adequacy in preload is often made and is used clinically every day in the operating room. Pulse pressure analysis is readily available from visual inspection or from monitoring used by the authors (GE S/5 Avance Anesthesia workstation) and is available at many centres. Trainees are instructed on how to interpret the PPV, such as to give intravascular fluid for the hypotensive patient when the PPV is high (i.e., $>13 \%$ ) and to treat with vasoactive agents when the PPV is low; especially in the fully ventilated surgical patient. Nevertheless, there is little evidence that supports the clinician or trainee on how to use this physiologic measure and there is no evidence that this will improve patient-related outcomes.

The authors used the "Grey Zone" approach first published by Cannesson et al. which is based on measuring PPV and cardiac output in a population undergoing cardiac, vascular, or digestive tract surgeries using various hemodynamic monitors. ${ }^{9}$ Positive responders were defined as an increase in cardiac output of $>15 \%$ in response to $500 \mathrm{~mL}$ of colloid solution (hetastarch $6 \%$ or modified gelatin) over 10-20 min. Using receiver operator curves, the authors conclude that a PPV of greater than $13 \%$ would likely respond with an increase in cardiac output. For values between $9 \%$ and $13 \%$, the response to fluids could not be reliably predicted, and, when less than $9 \%$, patients were unlikely to respond to fluid administration (i.e., they already had optimized preload). Kannan et al. modified this method by increasing the tidal volume from 8 to $12 \mathrm{~mL} \cdot \mathrm{kg}^{-1}$ for patients with a PPV between $9 \%$ and $13 \%$ : if the PPV increased to $\geq 17 \%$, fluid was administered to manage hypotension (defined by a systolic blood pressure $<90 \mathrm{~mm} \mathrm{Hg}$ or a mean arterial pressure (MAP) $<60 \mathrm{~mm} \mathrm{Hg}$ ); if the PPV was $<17 \%$, phenylephrine was administered. It should be recognized that there is no evidence to support this definition of hypotension. Furthermore, while the evidence may justify the use of PPV in the operating room to guide fluid administration, it is not sufficient to support the contention that fluid status was "optimized" as reported by Kannan et $a$. $^{8}$

Many may argue that blood pressure itself is not the determinant of DGF or acute kidney injury, but rather blood flow to the organ, and this may be correct. Apart from ultrasound-based assessment of renal blood flow, our ability to measure organ flow is limited. As a surrogate measure to blood flow, we consider MAP, the driving force for organ blood flow, as a target for our interventions. The evidence supporting the use of this surrogate measure is ever-increasing and this evidence is being used to define hypotension using MAP cutoffs and duration of hypotension as essential components of the definition. $^{10-12}$ Nevertheless, as the evidence of the detrimental effects of hypotension accrues, the definition of hypotension becomes more contentious. Often overlooked in the management of hypotension is the potential detrimental impact of the treatment themselves i.e., intravenous fluids, vasoactive agents, inotropes, chronotropes, or reduction in the depth of anesthesia. We must ask ourselves, is the treatment worse than the symptom?

In kidney transplantation, we have historically focused on fluid therapy for the management of hypotension. Kannan et al. have provided one potential strategy to avoid this (and only this) error. The evidence is clear: fluid overload is associated with poor outcomes and we must consider other strategies to manage hypotension, such as the use of dopexamine. ${ }^{13}$ There are numerous case reports and cohort studies of DGF and early graft loss in patients who already come to transplant with hypotension. ${ }^{14}$ The denervated transplant organ may be susceptible to the negative effects of hypotension, but the single-minded fluid-based approach to management may be even more detrimental. ${ }^{15}$ As Kannan et al. have made clear, we need to consider other therapies to manage hypotension.

Most importantly, postoperative care has a part in determining transplant outcomes. Optimizing intraoperative management will have little effect on kidney transplant outcome unless the fluid status is communicated to the postoperative care team and the optimization process is continued. If the patient has been optimized intraoperatively then immediately postoperatively, it makes little sense to respond to a reduction in urine output with fluid administration if the hemodynamics are unchanged.

As hypotension is associated with acute kidney injury in the non-transplant population, we believe hypotension may influence the risk of DGF in kidney 
transplants. Ultimately, improving the intraoperative hemodynamics and fluid management of kidney transplant patients may reduce the incidence of DGF and therefore improve the cost effectiveness of kidney transplantation. Using variables such as PPV may help to prevent fluid overload, but optimizing fluid management may require a more precise assessment of cardiac performance and the judicious use of fluids and/or cardiovascular medications in the entire perioperative period.

\section{La gestion liquidienne pour la transplantation rénale : s'agit-il vraiment d'une question de quantité?}

Chaque année, près de 1800 greffes de reins sont réalisées au Canada, mais malgré cet effort, le même nombre de personnes restent sur les listes d'attente pour une transplantation. ${ }^{1}$ Cette disparité dans l'offre et la demande d'organes est largement sous-estimée par le déficit réel : en effet, moins d'un cinquième des patients atteints d'insuffisance rénale terminale sont référés pour une transplantation dans les 12 mois suivant le début de leur dialyse. ${ }^{2}$ Parmi les greffes d'organes solides, la transplantation rénale est la plus rentable pour le système de santé, car elle offre les avantages les plus importants aux receveurs en termes de longévité et de qualité de vie. ${ }^{3} \mathrm{La}$ nécessité de la transplantation et ses avantages pour le receveur et notre système de santé constituent une incitation claire à nos efforts pour améliorer le fonctionnement du greffon de rein à court et à long terme. ${ }^{4}$

Dans la période qui suit immédiatement la transplantation, le retard de fonctionnement du greffon (RFG) est un phénomène courant, qui a été fortement associé à la durée du séjour hospitalier, aux complications postopératoires et au dysfonctionnement à long terme du greffon. ${ }^{5}$ Le retard de fonctionnement du greffon est généralement défini comme le besoin de dialyse dans la première semaine suivant la transplantation, mais il existe d'autres mesures du RFG fondées sur l'élimination de la créatinine après la transplantation ou sur les taux de réduction de la créatinine. Ces autres définitions pourraient être plus adaptées lorsque le besoin de dialyse est rare (p. ex., en cas de transplantation rénale d'un donneur vivant). ${ }^{6,} 7$ Quelle que soit la définition, un RFG survient dans environ 20 à $30 \%$ des greffes de rein de donneurs décédés et de 2 à $4 \%$ des greffes de rein de donneurs vivants. Alors que l'étiologie du RFG est multifactorielle, la prise en charge peropératoire est un facteur de risque potentiellement modifiable.

Dans ce numéro du Journal, Kannan et coll. comparent l'utilisation de la variabilité de la pression pulsée (VPP), mesurée à l'aide de la forme d'onde de monitorage artériel (poste de travail S/5 Avance ${ }^{\circledR}$ Anesthesia, GE Healthcare/ Datex-Ohmeda, Helsinki, Finlande), avec la pression veineuse centrale (PVC) pour guider l'administration liquidienne pendant la transplantation rénale. ${ }^{8}$ Les auteurs ont constaté une réduction notable de la quantité moyenne (écart type) de liquide administré dans le groupe VPP par rapport au groupe PVC (1346 [337] mL vs 1901 [379] mL). De toute évidence, la découverte de cette différence est déterminée autant par le groupe témoin que par le groupe de traitement de leur étude. Dans le groupe témoin, la PVC a été utilisée pour guider l'administration liquidienne sur la base de la méthode publiée par Othman et coll., dans laquelle les liquides sont restreints jusqu'à ce que le greffon de transplantation soit prêt pour l'anastomose vasculaire. À ce stade, la PVC est augmentée à $15 \mathrm{mmHg}$ avec l'administration de liquides intraveineux. ${ }^{4}$ Dans l'étude originale, cela a abouti à l'administration de plus de $3 \mathrm{~L}$ de sérum physiologique en salle d'opération. ${ }^{4}$

Comme le soulignent les auteurs, l'utilisation « traditionnelle » de la PVC pour guider la thérapie liquidienne est imparfaite en raison de « sa fiabilité limitée en tant que paramètre d'évaluation du statut liquidien intravasculaire ». L'objectif de l'anesthésiologiste est d'optimiser la précharge cardiaque ou, à tout le moins, de s'assurer que les performances cardiaques ou le volume d'éjection ne sont pas limités tout en évitant l'administration de quantités excessives de liquides. Les auteurs concluent qu'ils ont atteint cet objectif avec moins de liquides, mais que l'étude ne possédait pas suffisamment de puissance pour considérer toute issue cliniquement importante. Néanmoins, les hypothèses générées par cette étude devraient constituer la base de recherches futures utilisant soit une population plus importante, soit une population à haut risque de RFG.

Lorsqu'une forme d'onde artérielle est disponible, que ce soit à partir d'une canulation artérielle ou d'un monitorage non invasif (par exemple, ClearSight, Edwards Lifesciences, Irvine, CA, États-Unis), l'association physiologique entre variabilité de pression du pouls et adéquation de la précharge est souvent établie; en outre, elle est utilisée cliniquement tous les jours en salle d'opération. L'analyse de la pression du pouls est facilement disponible à partir d'une inspection visuelle ou du monitorage utilisé par les auteurs (poste de travail GE $\mathrm{S} / 5$ Avance Anesthesia) et est disponible dans de nombreux centres. On enseigne aux résidents la façon d'interpréter la VPP, par exemple en administrant des liquides intravasculaires aux patients hypotendus lorsque leur VPP 
est élevée (c.-à-d. > $13 \%$ ) et en donnant des agents vasoactifs lorsque la VPP est basse - en particulier chez le patient opéré sous ventilation contrôlée. Néanmoins, il existe peu de données probantes appuyant le clinicien ou le résident quant à la façon d'utiliser cette mesure physiologique, et il n'existe aucune donnée probante attestant qu'elle améliorera les issues liées aux patients.

Les auteurs ont utilisé l'approche de «zone grise » publiée pour la première fois par Cannesson et coll., qui se fonde sur la mesure de la VPP et du débit cardiaque dans une population subissant des chirurgies cardiaques, vasculaires ou digestives avec utilisation concomitante de divers moniteurs hémodynamiques. ${ }^{9}$ Les répondants positifs ont été définis comme ceux affichant une augmentation du débit cardiaque de $>15 \%$ en réponse à $500 \mathrm{~mL}$ de solution colloïdale (hétamidon $6 \%$ ou gélatine modifiée) sur une période de 10 à $20 \mathrm{~min}$. En utilisant des courbes ROC (receiver operator curves), les auteurs concluent qu'une VPP supérieure à $13 \%$ répondrait probablement avec une augmentation du débit cardiaque. Pour les valeurs comprises entre $9 \%$ et $13 \%$, la réponse aux liquides ne pouvait pas être prédite de manière fiable et, lorsqu'elle était inférieure à $9 \%$, il était peu probable que les patients réagissent à l'administration de liquides (autrement dit, leur précharge était déjà optimisée). Kannan et coll. ont modifié cette méthode en augmentant le volume courant de 8 à $12 \mathrm{~mL} \cdot \mathrm{kg}^{-1}$ pour les patients ayant une VPP comprise entre $9 \%$ et $13 \%$ : si la VPP augmentait à $\geq 17$ $\%$, des liquides étaient administrés pour prendre en charge l'hypotension (définie par une tension artérielle systolique $<90 \mathrm{mmHg}$ ou une tension artérielle moyenne (TAM) $<$ $60 \mathrm{mmHg}$ ); si la VPP était $<17 \%$, de la phényléphrine était administrée. Il convient de mentionner qu'il n'existe aucune donnée probante à l'appui de cette définition de l'hypotension. De plus, bien que les données probantes puissent justifier l'utilisation de la VPP en salle d'opération pour orienter l'administration liquidienne, cela ne suffit pas pour étayer l'affirmation selon laquelle l'état liquidien a été «optimisé », tel que rapporté par Kannan et coll. ${ }^{8}$

Plusieurs pourraient soutenir l'idée que la tension artérielle en soi ne peut être le déterminant d'un RFG ou d'une insuffisance rénale aiguë, mais plutôt que ce serait le flux sanguin vers l'organe - ce qui pourrait être correct. Hormis l'évaluation par ultrasons de la circulation sanguine rénale, notre capacité à mesurer la circulation dans les organes est limitée. En tant que mesure de substitution de la circulation sanguine, nous considérons la TAM, la force motrice du flux sanguin aux organes, comme une cible pour nos interventions. Les données probantes appuyant l'utilisation de cette mesure de substitution sont de plus en plus nombreuses, et ces données sont utilisées pour définir l'hypotension en utilisant les seuils de TAM et la durée de l'hypotension comme composantes essentielles de la définition. ${ }^{10-12}$ Néanmoins, à mesure que les données probantes étayant les effets néfastes de l'hypotension s'accumulent, sa définition devient de plus en plus controversée. L'impact négatif potentiel du traitement lui-même est souvent négligé dans la prise en charge de l'hypotension, qu'il s'agisse de liquides intraveineux, d'agents vasoactifs, d'inotropes, de chronotropes ou d'une réduction de la profondeur de l'anesthésie. Nous devons nous poser la question : le traitement est-il pire que le symptôme?

En transplantation rénale, nous nous sommes historiquement concentrés sur la thérapie liquidienne pour la prise en charge de l'hypotension. Kannan et coll. proposent une stratégie potentielle pour éviter cette erreur (et seulement celle-ci). Les données probantes sont sans équivoque : la surcharge liquidienne est associée à de mauvaises issues et nous devons envisager d'autres stratégies pour prendre en charge l'hypotension, telles que l'utilisation de la dopexamine. ${ }^{13}$ Il existe de nombreuses présentations de cas et études de cohorte sur le RFG et la perte précoce du greffon chez les patients se présentant pour une greffe en souffrant d'une hypotension existante. ${ }^{14}$ L'organe transplanté dénervé peut être sensible aux effets négatifs de l'hypotension, mais une approche se fondant uniquement sur les fluides pourrait être encore plus préjudiciable. ${ }^{15}$ Comme Kannan et coll. l'ont clairement indiqué, nous devons envisager d'autres thérapies pour prendre en charge l'hypotension.

Plus important encore, les soins postopératoires ont un rôle à jouer dans les issues de la transplantation. L'optimisation de la prise en charge peropératoire aura peu d'effet sur l'issue de la greffe de rein à moins que l'état liquidien ne soit communiqué à l'équipe de soins postopératoires et que le processus d'optimisation ne se poursuive. Si le patient a été optimisé en peropératoire puis immédiatement après l'opération, il serait illogique de répondre à une réduction de la diurèse en administrant des fluides lorsque les valeurs hémodynamiques demeurent inchangées.

Comme l'hypotension est associée à l'insuffisance rénale aiguë dans la population générale (c.-à-d. non transplantée), nous pensons que l'hypotension pourrait influencer le risque de RFG dans les greffes de rein. En fin de compte, l'amélioration de l'hémodynamie peropératoire et de la gestion liquidienne des patients de transplantation rénale pourrait réduire l'incidence de RFG et donc améliorer la rentabilité de la transplantation rénale. L'utilisation de variables telles que la VPP pourrait aider à prévenir la surcharge liquidienne, mais l'optimisation de la gestion liquidienne pourrait nécessiter une évaluation plus précise des performances cardiaques et l'utilisation judicieuse de liquides et / ou de médicaments cardiovasculaires pendant toute la période périopératoire. 
Disclosures None.

\section{Funding statement None.}

Editorial responsibility This submission was handled by Dr. Philip M. Jones, Deputy Editor-in-Chief, Canadian Journal of Anesthesia,

Déclaration Aucune.

Déclaration de financement Aucune.

Responsabilité éditoriale Cet article a été traité par Dr Philip M. Jones, rédacteur en chef adjoint, Journal canadien d'anesthésie.

\section{References}

1. Canadian Institute for Health Information. Annual statistics on Organ Replacement in Canada. Dialysis, Transplantation and Donation; 2010 to 2019. Available from URL: https://www.cihi. $\mathrm{ca} /$ sites/default/files/document/corr-dialysis-transplantationdonation-2010-2019-snapshot-en.pdf (accessed September 2021).

2. Kim SJ, Gill JS, Knoll G, et al. Referral for kidney transplantation in Canadian provinces. J Am Soc Nephrol 2019, 30: 1708-21.

3. Tonelli M, Wiebe $N$, Knoll $G$, et al. Systematic review: kidney transplantation compared with dialysis in clinically relevant outcomes. Am J Transplant 2011; 11: 2093-109.

4. Othman MM, Ismael AZ, Hammouda GE. The impact of timing of maximal crystalloid hydration on early graft function during kidney transplantation. Anesth Analg 2010; 110: 1440-6.

5. Yarlagadda SG, Coca SG, Formica RN Jr, Poggio ED, Parikh $C R$. Association between delayed graft function and allograft and patient survival: a systematic review and meta-analysis. Nephrol Dial Transplant 2009, 24: 1039-47.

6. Wu WK, Famure $O, \operatorname{Li} Y$, Kim SJ. Delayed graft function and the risk of acute rejection in the modern era of kidney transplantation. Kidney Int 2015; 88: 851-8.
7. Yarlagadda $S G$, Coca $S G$, Garg $A X$, et al. Marked variation in the definition and diagnosis of delayed graft function: a systematic review. Nephrol Dial Transplant 2008; 23: 2995-3003.

8. Kannan $G$, Loganathan $S$, Kajal $K$, et al. The effect of pulse pressure variation compared to central venous pressures on intraoperative fluid management during kidney transplant surgery: a randomized controlled trial. Can J Anesth 2022; DOI: https://doi.org/10.1007/s12630-021-02130-y.

9. Cannesson M, Le Manach Y, Hofer CK, et al. Assessing the diagnostic accuracy of pulse pressure variations for the prediction of fluid responsiveness: a "gray zone" approach. Anesthesiology 2011; 115: 231-41.

10. Sun LY, Wijeysundera DN, Tait GA, Beattie WS. Association of intraoperative hypotension with acute kidney injury after elective noncardiac surgery. Anesthesiology 2015; 123: 515-23.

11. Gregory A, Stapelfeldt WH, Khanna AK, et al. Intraoperative hypotension is associated with adverse clinical outcomes after noncardiac surgery. Anesth Analg 2021; 132: 1654-65.

12. Walsh M, Devereaux PJ, Garg AX, et al. Relationship between intraoperative mean arterial pressure and clinical outcomes after noncardiac surgery: toward an empirical definition of hypotension. Anesthesiology 2013; 119: 507-15.

13. Pearse RM, Harrison DA, MacDonald $N$, et al. Effect of a perioperative, cardiac output-guided hemodynamic therapy algorithm on outcomes following major gastrointestinal surgery: a randomized clinical trial and systematic review. JAMA 2014; 311: 2181-90.

14. Alhamad T, Brennan DC, Brifkani Z, et al. Pretransplant midodrine use: a newly identified risk marker for complications after kidney transplantation. Transplantation 2016; 100: 1086-93.

15. Challand $C$, Struthers $R$, Sneyd JR, et al. Randomized controlled trial of intraoperative goal-directed fluid therapy in aerobically fit and unfit patients having major colorectal surgery. $\mathrm{Br} \mathrm{J}$ Anaesth 2012; 108: 53-62.

Publisher's Note Springer Nature remains neutral with regard to jurisdictional claims in published maps and institutional affiliations. 